\title{
IL CONGIUNTIVO NELL'ITALIANO SCRITTO E PARLATO
}

\author{
Eva Klímová \\ Università della Slesia, Opava
}

\begin{abstract}
The article deals with the usage of the subjunctive in contemporary Italian against the background of the grammatical rules that determine its usage in standard Italian. The subjunctive is a phenomenon widely discussed and sometimes viewed as dying in spoken Italian because of difficulties that even Italian users meet when learning its forms. Still the usage of the subjunctive permits the speaker to express small differences in modal meanings within both deontic and epistemic modality. At the same time, it is considerate as a perfect and elegant stylistic means that allows the speaker to express different attitudes in regards to what he or she is saying. The analysis is based on the LIP corpus.
\end{abstract}

Key words : Subjunctive; written and spoken Italian; deontic; epistemic; modality

\section{Introduzione}

Questo contributo si propone lo scopo di esaminare l'uso del congiuntivo italiano nella lingua scritta e in quella parlata per cercare di dimostrare se davvero esso sia "condannato a morire", essendo usato, come dicono parecchi italiani, sempre di meno nella conversazione quotidiana. Nel ventennio successivo alla Seconda guerra mondiale era possibile definire le differenze tra la lingua scritta e la lingua parlata. I testi scritti erano più elaborati, influenzati dai modelli letterari e da norme grammaticali applicate con coerenza. Oggi le differenze tra le due varietà linguistiche sembrano spiccare di meno e la frontiera tra di loro si fa sempre meno netta, fatto dovuto probabilmente all'uso delle tecnologie della comunicazione apparse negli ultimi venti anni. Da una parte infatti si colloca la varietà usata ad esempio durante una telefonata, gli "small talk" di tutti i giorni, i programmi televisivi come i diversi dibattiti di economia e di politica la cui lingua sicuramente sarà diversa da quella dei talk show o dei varietà, benché in tutti quanti si senta quella che è considerabile come lingua parlata, quella "dell'uso medio"1. Dall'altra parte si trova la varietà della lingua dei giornali, dei saggi scientifici e quella della narrativa, che rappresentano lingua scritta.

Anche se nell'italiano parlato d'oggi si osserva un certo abbandono dell'uso del congiuntivo a tal punto che si parla della sua "perdita di terreno", esso rimane vivo ed è, in molte sue funzioni, uno strumento molto produttivo e insostituibile. A proposito del pericolo della perdita di terreno del congiuntivo sono degni di nota non solo i risultati presentati nel saggio di Lombardi Vallauri (si veda nota 1) ma anche quelli della ricerca presentata recentemente da una studiosa polacca: analizzando il corpus dello stile giornalistico, che è molto vicino alla lingua parlata, ha osservato frasi con alcune espressioni modali nella frase principale, dividendone le espressioni in tre gruppi: espressioni di significato volitivo, dubitativo e valutativo che richiedono o ammettono l'uso del congiuntivo nella proposizione dipendente, in cui cioè il congiuntivo è obbligatorio o facoltativo,

\footnotetext{
${ }^{1}$ MARAZZINI (2004: 217).

${ }^{2}$ Cfr. LOMBARdi VAllaURi (2003: 609), con riferimento ad altre fonti.

Echo des études romanes XI/2, 2015 
arrivando all'osservazione che il congiuntivo "non è morto e non sembra neppure destinato a sparire" ${ }^{\text {. }}$.

Dal momento che vogliamo occuparci del congiuntivo, uno dei modi verbali, bisogna, almeno brevemente e a grandi linee, soffermarsi sulla funzione del modo verbale nell'ambito della modalità definita come "grammaticalisation of speaker's (subjective) attitudes and opinions" ${ }^{\text {"4 }}$. Essendo una delle categorie grammaticali del verbo, serve a indicare diversi significati modali sullo schema dei tipi di frase, e cioè della frase dichiarativa, interrogativa, iussiva e ottativa contribuendo non solo all'indicazione di diversi tipi di atto illocutorio ma anche all'indicazione di altri tipi di modalità: quella epistemica e quella deontica. Da questo punto di vista il modo verbale rappresenta uno strumento difficilmente sostituibile. La frase:

\section{Mi daresti una mano?}

dal punto di vista della forma, è una interrogativa classificabile come domanda, che può essere pronunciata dal parlante con lo scopo di indurre l'interlocutore ad eseguire un' azione, quindi è considerabile come richiesta, alla quale il condizionale dà una sfumatura di maggiore cortesia. Quindi il condizionale daresti serve da strumento per esprimere, anche se in modo indiretto, la volontà del parlante ed è definibile come modo deontico. Allo stesso tempo con il condizionale il parlante esprime una certa insicurezza sulla proposizione, quindi esso può essere considerato un modo epistemico.

Come evidenzia l'esempio precedente, uno stesso enunciato è associabile non solo con alcuni atti illocutori, ma anche con diversi tipi di modalità, cioè sia con la modalità deontica sia con quella epistemica. È per questa ragione che il modo verbale può essere considerato uno strumento polifunzionale e quindi uno strumento implicito della modalità dell'enunciato.

Se il modo verbale è interpretabile come strumento implicito dell'espressione dell' atteggiamento del parlante, consideriamo come modo esplicito un insieme di elementi modali nella frase complessa: in essa il verbo predicativo della frase principale funge da modus, e la proposizione dipendente da dictum 5 .

Nella frase complessa, la proposizione principale e quella dipendente sono unite non solo da un legame sintattico. Tra di loro intercorre anche una relazione semantica, cioè modale. A evidenziarlo è il fatto che in italiano è il significato modale del verbo predicativo della proposizione principale a determinare il modo del verbo della proposizione dipendente. Così la doppia dipendenza, quella sintattica e quella semantica, si manifesta come una realizzazione della categoria modale.

Qui di seguito si parlerà dapprima del congiuntivo italiano nella lingua scritta e successivamente in quella parlata, concentrandosi sul suo uso nella proposizione dipendente e lasciando invece in disparte la sua funzione nella frase principale ${ }^{6}$. C'è un semplice motivo: nella frase complessa, il parlante, dopo certe espressioni

\footnotetext{
${ }^{3}$ Cfr. SIERON (2013), con riferimento a WANDRUSZKA (1991).

${ }^{4}$ PALMER (1986:16).

${ }^{5}$ Cfr. Alisova (1972:163), con riferimento a Bally (1963).

${ }^{6}$ Sul congiuntivo obbligatorio nelle proposizioni dipendenti cfr. WANDRUSZKA (1991). 
modali presenti nella frase principale, può scegliere tra il congiuntivo e l'indicativo nella proposizione dipendente, mentre nella frase semplice il parlante non si trova di fronte ad un dilemma del genere. Basti menzionare due casi in cui la lingua italiana non permette altro che il congiuntivo: il primo riguarda la forma della terza persona del congiuntivo presente nelle frasi iussive quando il parlante si rivolge a una persona dandole del Lei; in questo caso il congiuntivo serve per esprimere un certo grado di cortesia o di formalità fungendo da imperativo nelle richieste formali, di conseguenza il suo uso è legato piuttosto alla manifestazione dei principi della conversazione, tra cui spicca quello della cortesia. L'altro caso riguarda il congiuntivo imperfetto e il congiuntivo piuccheperfetto, insostituibili nelle frasi ottative.

Le osservazioni legate all'uso del congiuntivo nella lingua parlata si basano sull'analisi di un corpus linguistico, e sono presentate assieme alla descrizione del corpus e del metodo.

\section{Il congiuntivo nell'italiano scritto}

Le norme determinanti per l'uso del modo verbale nella proposizione dipendente, la cui applicazione è osservabile nella lingua scritta, deriverebbero dalla classificazione delle espressioni modali nella proposizione reggente. Per l'italiano ne sono state proposte cinque classi ${ }^{7}$ :

Verba dicendi ("verbi assertivi forti"): assicurare, dire, raccontare ecc.

Verba percipiendi ("verbi di percezione"): vedere, sentire, osservare, rendersi conto ecc.)

Verba sentiendi ("verbi assertivi deboli"): pensare, credere, immaginare, capire, ritenere ecc.)

Verbi di volontà: ordinare, volere, desiderare, chiedere ecc.

Verbi emotivi: (dis)piacere, stupirsi, offendersi ecc.

Dopo i verbi della prima e della seconda classe, cioè dopo i verba dicendi e $\mathrm{i}$ verba percipiendi, in una proposizione dipendente in italiano si possono usare sia l'indicativo che il condizionale. Con l'indicativo, l'azione della proposizione completiva è reale, con il condizionale solo possibile. In altre parole, l'indicativo presenta l'azione come un fatto e quindi funge da modo epistemico indicabile come factive, il condizionale invece come non-factive ${ }^{8}$. Nell' enunciato:

Dico che ne sei responsabile.

il significato del verbo assertivo forte dire della frase principale è il modus che assieme all'indicativo sei di quella dipendente sono i partecipanti ad un modo analitico esplicito considerabile come modo assertivo.

\footnotetext{
${ }^{7}$ Cfr. per esempio BronZi (1977: 431-432), e SCHNEIDER (1999: 56). Cfr. inoltre KLímová (2008), dove si fa riferimento alla stessa fonte.

${ }^{8}$ Cfr. LyONS (1977: 794-795).
} 
Dopo i verbi della terza, della quarta e della quinta classe, cioè dopo i verba sentiendi, dopo i verbi di volontà e dopo i verbi emotivi, le norme grammaticali richiedono, nell'italiano standard, il congiuntivo. Nell' enunciato:

Credo/Dubito che tu sia responsabile.

i verbi credere e dubitare, appartenenti alla classe dei "verbi assertivi deboli", esprimono un dubbio o un grado di sicurezza del parlante, e il congiuntivo nella proposizione dipendente ha la funzione di non-factive epistemico. Appartengono a questa classe di espressioni modali i predicativi comprendenti un aggettivo come $\grave{e}$ (im)possibilelè (im)probabile, che, essendo strutture impersonali, offrono al parlante la possibilità di esprimere un distacco dall'affermazione. Il significato del verbo assertivo debole come credere o dubitare della frase principale assieme al congiuntivo di quella dipendente sono i partecipanti ad un modo analitico esplicito considerabile come modo dubitativo. La frase:

Voglio/Desidero che tu sia responsabile.

rappresenta un nuovo schema modale. Nella frase principale dello schema sono osservabili espressioni modali di volontà. Quindi il dictum della proposizione dipendente è presentato come voluto, aspettato, richiesto, e cioè ancora non-reale, che deve ancora avverarsi. Il soggetto modale, il parlante, indica come desiderata, necessaria o richiesta la realizzazione dell'azione, ed il congiuntivo, che è obbligatorio nella proposizione dipendente, contribuisce all'espressione di un significato nell'ambito della modalità deontica. È considerabile una variante di questo schema una struttura con il verbo sperare in un enunciato come: Spero che tu ti comporti da persona responsabile. Infatti, il parlante può usarlo per esprimere: Voglio/Desidero che tu ti comporti da persona responsabile. Fanno parte delle espressioni modali di questa classe anche i predicati impersonali bisogna/occorre, che indicano l'azione come necessaria o opportuna, con il significato cioè di necessità deontica. Il significato del verbo di volontà come volere o desiderare della frase principale assieme al congiuntivo di quella dipendente sono i partecipanti ad un modo analitico esplicito considerabile come modo imperativo. Vediamo lo schema modale successivo:

Mi (dis)piace che tu (non) sia responsabile.

La frase principale di questo schema contiene i verbi piacere o dispiacere con i quali il parlante esprime un atteggiamento della valutazione positiva o di quella negativa nei confronti del contenuto della proposizione. Fanno parte di questa classe i predicati impersonali come per esempio (non) è giusto. Nella proposizione viene usato, anche se non esclusivamente, il congiuntivo che, assieme all'espressione modale della frase principale, è un coefficiente del modo analitico esplicito che possiamo denominare modo valutativo?

9 Per la lista delle espressioni modali valutative e la loro reggenza modale cfr. per es. SCHNEIDER (1999:171-180). 


\section{Il congiuntivo nell'italiano parlato}

\subsection{Il corpus e metodo}

Per la nostra analisi abbiamo usato il corpus LIP (Lessico di frequenza dell'italiano parlato), una delle collezioni di testi più importanti della lingua italiana parlata comprendente circa 490 mila parole, esaminando il modo verbale della proposizione dipendente dopo determinate espressioni modali nella frase principale. Anche se il LIP è un corpus non particolarmente aggiornato e quindi non riflette necessariamente il parlato di oggi, utilizzandolo si può compiere un'analisi sulla base di una tipologia del discorso che sembra adatta per i nostri scopi, considerando che comprende cinque gruppi di registrazioni, indicate con le lettere A, B, C, D, E. Le registrazioni dei gruppi A, B e C sono composte da conversazioni bidirezionali. Il gruppo A da quelle in casa, quelle avvenute in ambito universitario o quelle sui mezzi di trasporto; il gruppo B da conversazioni telefoniche; il gruppo $\mathrm{C}$ dalle conversazioni più complesse, quali conversazioni delle assemblee legislative, dei dibattiti culturali, degli incontri di lavoro o degli esami universitari. Rappresentano il gruppo D i discorsi unidirezionali (quali le lezioni scolastiche, le lezioni universitarie, le relazioni in convegni scientifici); e infine il gruppo E, che comprende gli scambi comunicativi unidirezionali a distanza (tipo le trasmissioni televisive o radiofoniche) ${ }^{10}$. Le registrazioni sono state fatte in quattro città italiane: Milano (M), Firenze (F), Roma (R) e Napoli $(\mathrm{N})$. Il genere e il luogo di registrazione sono indicati accoppiando le lettere iniziali: NB indica una conversazione del gruppo B registrata a Napoli. Come si vede, la tipologia dei discorsi comprende molte varietà sia diamesiche, che diafasiche e diatopiche. Una varietà così ampia obbliga a trarre dalle analisi conclusioni generali: è quasi impossibile fare delle distinzioni sulla base di tutti i criteri di suddivisione della raccolta.

La classificazione delle espressioni modali fornita qui sopra serve da punto di partenza nelle nostre analisi, , lasciando in disparte i verbi assertivi forti e i verba percipiendi, perché dopo di essi, univocamente, viene usato l'indicativo, e individuando, dopo una scrupolosa scelta, $i$ verbi della terza, della quarta e della quinta classe: quelli che esprimono un'opinione soggettiva del parlante (credere, dubitare, immaginare, pensare, ritenere, sperare); quelli che esprimono volontà e desiderio (volere, desiderare); i verbi emotivi che esprimono sensazioni (meravigliarsi, essere contento, avere paura/timore, dispiacersi). In una proposizione oggettiva retta da essi, questi verbi, in italiano standard, richiedono, il congiuntivo, anche se con alcuni non è escluso l'indicativo ${ }^{11}$. Inoltre sono state individuate e prese in considerazione anche le strutture impersonali è bene, $\grave{e}$ giusto, è opportuno, è possibile, è normale, e i verbi impersonali bisogna, pare,

\footnotetext{
10 Sui problemi della tipologia comunicativa e sull'eterogeneità dei gruppi vedi SCHNEIDER (1999:36), con riferimento a P. Koch (1994), Prime esperienze con i corpora LIP, in T. De Mauro (a cura di), Come parlano gli italiani, La Nuova Italia, Firenze, pp. 201-2016.
}

${ }^{11}$ Cfr. WANDRUSZKA (1991:415-481). 
sembra. Queste espressioni fungono da predicati modali quando reggono una proposizione soggettiva e richiedono pure essi il congiuntivo.

\subsection{L'analisi}

Nel corso dell'analisi sono state individuate nella frase principale solo le forme verbali della prima e della seconda persona singolare perché queste forme indicano gli interlocutori del discorso: la persona che parla e quella che ascolta. Indicano cioè i diretti partecipanti alla situazione comunicativa, che esprimono il proprio atteggiamento verso la proposizione. Rimangono fuori dall'esame le forme dei verbi del tipo chiedersi, domandarsi, che introducono una domanda indiretta: essi non richiedono obbligatoriamente l'uso del congiuntivo. ${ }^{12}$ Come accennato sopra, alcuni verbi ed espressioni modali permettono, al parlante, di scegliere tra il congiuntivo e l'indicativo. Il motivo per cui non sono state prese in considerazione esclusivamente le espressioni che obbligatoriamente richiedono il congiuntivo è semplice: esaminare l'uso del congiuntivo solo nei contesti in cui viene escluso l'indicativo vorrebbe dire mostrare solo una faccia della medaglia. In altre parole, l'analisi non risponderebbe alla domanda se il congiuntivo nella lingua parlata venga evitato o meno ${ }^{13}$. Non sono state incluse nel conteggio le forme della prima persona plurale dei verbi della proposizione dipendente perché non sono morfologicamente identificabili come forme del congiuntivo. Successivamente verranno presentati i risultati dell'indagine partendo dal modo verbale della proposizione oggettiva e passando a quello della proposizione soggettiva. Per ogni categoria viene dato un esempio preso dal LIP con una sigla che indica la parte del corpus.

\subsubsection{L'uso del congiuntivo nella proposizione oggettiva}

Sono state individuate, nel corpus, strutture modali predicative che richiedono o permettono, nella proposizione oggettiva, il congiuntivo:

La tabella 1 evidenzia l'uso del modo dopo le espressioni modali che esprimono un'opinione soggettiva, un dubbio o l'incertezza del parlante: credere, dubitare, immaginare, pensare, ritenere, sperare

\footnotetext{
${ }^{12}$ Cfr. WANDRUSZKA (1991:471).

${ }^{13}$ Cfr. una simile osservazione in LOMBARDI VALLAURI (2003:612). 


\begin{tabular}{|c|c|c|c|c|c|c||}
\hline MODO & credo & $\begin{array}{c}\text { penso } \\
\text { pensi } \\
\text { pensiamo }\end{array}$ & immagino & $\begin{array}{c}\text { ritengo } \\
\text { riteniamo }\end{array}$ & spero & dubito \\
\hline Totale occorrenze & 149 & $84(73 / 6 / 5)$ & 2 & $21(14 / 7)$ & 12 & 1 \\
\hline Congiuntivo & 95 & $52(47 / 3 / 2)$ & 2 & $16(11 / 5)$ & 10 & 1 \\
\hline Indicativo & 36 & $22(18 / 2 / 2)$ & 0 & $5(3 / 2)$ & 1 & - \\
\hline Futuro/Condizionale & $6 / 12$ & $10(8 / 1 / 1)$ & 0 & 0 & 1 & - \\
\hline \multicolumn{7}{|c|}{ Percentuale } \\
\hline Congiuntivo Indicativo & $\mathbf{6 4} \%$ & $\mathbf{6 2} \%$ & $\mathbf{1 0 0 \%}$ & $\mathbf{7 6} \%$ & $\mathbf{8 3} \%$ & $\mathbf{1 0 0} \%$ \\
Futuro/Condizionale & $\mathbf{2 4} \%$ & $\mathbf{2 5} \%$ & $\mathbf{0 \%}$ & $\mathbf{2 4} \%$ & $\mathbf{8 , 5} \%$ & $\mathbf{0} \%$ \\
$\mathbf{1 2 \%}$ & $\mathbf{1 3 \%}$ & $\mathbf{0 \%}$ & $\mathbf{0 \%}$ & $\mathbf{8 , 5} \%$ & $\mathbf{0} \%$ \\
\hline
\end{tabular}

Tabella 1

Nel corpus sono state individuate 167 strutture con credo di cui solo 149 sono state prese in considerazione. Nei 18 casi rimanenti il parlante aveva interrotto il discorso senza continuare a parlare. Dopo credo il congiuntivo è stato individuato nel $64 \%$ delle occorrenze, dopo penso/pensi/pensiamo la percentuale a suo favore è stata del $62 \%$, dopo ritengo/riteniamo il congiuntivo è stato usato nel $76 \%$ dei casi e dopo spero la percentuale è dell' $83 \%$. Solo il congiuntivo è stato usato dopo immagino (2 occorrenze) e dopo dubito (1 occorrenza). Quindi si può concludere che anche se il congiuntivo è sostituibile dall'indicativo nelle strutture in cui funge da coefficiente del modo epistemico analitico, anche nella lingua parlata gli viene accordata preferenza. Vediamo un esempio:

... io spero che sia solo stanchezza e quindi ...LIP FB11

Il modo dopo le espressioni modali della volontà e del desiderio volere, richiedere, bisogna, è necessario:

\begin{tabular}{||c|c|c|c|c||}
\hline & richiedere & $\begin{array}{c}\text { voglio } \\
\text { vogliamo }\end{array}$ & bisogna & è necessario \\
\hline Totale & 1 & 2 & 10 & 1 \\
\hline Congiuntivo & 1 & 2 & 7 & 1 \\
\hline Indicativo & 0 & 0 & 3 & 0 \\
\hline \multicolumn{5}{|c||}{ Percentuale } \\
\hline $\begin{array}{c}\text { Congiuntivo } \\
\text { Indicativo }\end{array}$ & $\begin{array}{c}\mathbf{1 0 0} \% \\
\mathbf{0} \%\end{array}$ & $\begin{array}{c}\mathbf{1 0 0} \% \\
\mathbf{0} \%\end{array}$ & $\begin{array}{c}\mathbf{7 0} \% \\
\mathbf{3 0} \%\end{array}$ & $\begin{array}{c}\mathbf{1 0 0} \% \\
\mathbf{0} \%\end{array}$ \\
\hline
\end{tabular}

Tabella 2

Nel corpus sono state individuate poche strutture con questi predicati modali: una volta la struttura con il verbo richiedere e due volte quella con il verbo volere nella frase principale. Come si vede, è possibile trovare solo il congiuntivo nelle strutture in cui esso funge da coefficiente del modo deontico analitico. Sono state incluse in questa categoria anche le espressioni modali impersonali bisogna ed ̀̀ necessario, sebbene esse possano essere facilmente incluse nella categoria delle 
espressioni valutative. La bassa frequenza delle espressioni della volontà e del desiderio è dovuta, a nostro avviso, al fatto che, nella lingua parlata, alle strutture di una frase complessa viene preferito l'uso della frase semplice, ossia la frase iussiva all'imperativo o al congiuntivo esortativo, perché rappresentano un modo diretto per esprimere la richiesta, l'ordine o il permesso. Per esempio:

Voglio che lei mi faccia capire che ... $\quad$ LIP MC11

Il modo dopo le espressioni modali che esprimono sensazioni: meravigliarsi, essere contento, avere paura (timore), dispiacersi;

\begin{tabular}{||c|c|c|c|c||}
\hline \hline & meravigliarsi & essere contento & $\begin{array}{c}\text { avere paura } \\
\text { (timore) }\end{array}$ & dispiacere \\
\hline Totale & 1 & 3 & 4 & 3 \\
\hline Congiuntivo & 1 & 0 & 4 & 1 \\
\hline Indicativo & 0 & 3 & 0 & 2 \\
\hline \multicolumn{5}{|c||}{ Percentuale } \\
\hline $\begin{array}{c}\text { Congiuntivo } \\
\text { Indicativo }\end{array}$ & $\mathbf{1 0 0 \%}$ & $\mathbf{0 \%} \%$ & $\begin{array}{c}\mathbf{1 0 0} \% \\
\mathbf{0 \%}\end{array}$ & $\mathbf{3 3} \%$ \\
\hline
\end{tabular}

Tabella 3

Anche con queste espressioni sono state individuate, nella frase principale del corpus, poche occorrenze: una volta con il verbo meravigliarsi, due volte con il predicato modale essere contento, quattro volte con la struttura predicativa avere paura o timore, e tre volte con il verbo dispiacersi; come dimostra la tabella 3 , nella maggior parte dei casi è stato usato il congiuntivo nella proposizione dipendente: esso resiste, anzi prevale dopo meravigliarsi e dopo avere paura, dopo il verbo dispiacersi invece prevale l'indicativo (66 \%). Tuttavia si può constatare che la notevole prevalenza del congiuntivo dopo i predicati che permettono l'uso dell'indicativo è la testimonianza della vivacità del primo. Anche in questo caso offriamo qualche esempio:

... io mi meraviglio che non sia già stato fatto ... $\quad$ LIP NC4

... mi dispiace che non c'è il sindaco ma ... LIP RC1

\subsubsection{L'uso del congiuntivo nella proposizione soggettiva}

Sono state individuate, nel corpus, strutture modali predicative che richiedono o permettono, nella proposizione soggettiva, il congiuntivo:

Il modo dopo le espressioni impersonali costituite da una forma del verbo essere unita a un aggettivo o ad un sostantivo, quali è bene, è giusto, è opportuno, $\grave{e}$ possibile, è normale ${ }^{14}$. In questa categoria sono stati inclusi i verbi impersonali pare e sembra:

\footnotetext{
${ }^{14}$ Anche qui si può confrontare con i risultati attestati dalla SIEROŃ (2013).
} 


\begin{tabular}{|c|c|c|c|c|c|c|c|}
\hline & $\begin{array}{c}\grave{e} \\
\text { bene }\end{array}$ & $\begin{array}{c}\grave{e} \\
\text { giusto }\end{array}$ & $\begin{array}{c}\grave{e} \\
\text { opportuno }\end{array}$ & $\begin{array}{c}\grave{e} \\
\text { possibile }\end{array}$ & $\begin{array}{c}\grave{e} \\
\text { normale }\end{array}$ & pare & sembra \\
\hline Totale & 3 & 10 & 2 & 7 & 2 & 51 & 45 \\
\hline Congiuntivo & 3 & 10 & 2 & 4 & 2 & 29 & 32 \\
\hline Indicativo & 0 & 0 & 0 & 3 & 0 & 22 & 13 \\
\hline \multicolumn{8}{|c|}{ Percentuale } \\
\hline $\begin{array}{l}\text { Congiuntivo } \\
\text { Indicativo }\end{array}$ & $\begin{array}{c}100 \\
\% \\
0 \%\end{array}$ & $\begin{array}{c}100 \% \\
0 \%\end{array}$ & $\begin{array}{c}100 \% \\
0 \%\end{array}$ & $\begin{array}{l}53 \% \\
47 \%\end{array}$ & $\begin{array}{c}100 \% \\
0 \%\end{array}$ & $\begin{array}{l}57 \\
\% \\
43 \\
\%\end{array}$ & $\begin{array}{l}71 \% \\
29 \%\end{array}$ \\
\hline
\end{tabular}

Tabella 4

Come si vede nella tabella, dopo quasi tutte le espressioni di questa categoria i parlanti hanno usato quasi esclusivamente il congiuntivo, con l'eccezione della struttura è possibile. Anche con i verbi impersonali prevale il congiuntivo, anzi con sembra la sua prevalenza è notevole:

\footnotetext{
... se è possibile che ci sia un impegno LIP NC4 miseria...

... quindi mi pare che anche da questo punto di vista non si possa continuare a piangere LIP ME6
}

\section{Conclusione}

Il congiuntivo, in italiano, è un fenomeno largamente discusso, considerato addirittura come condannato a morire nella lingua parlata. Tuttavia, con il congiuntivo si ha a disposizione un ulteriore strumento linguistico rispetto alle lingue che non ne dispongono e che si avvalgono di strumenti lessicali. Esso dà al parlante la possibilità di esprimere esplicitamente che quello che dice è una sua opinione soggettiva, e quindi si ha la possibilità di mantenere un distacco dalla proposizione. Nel corpus usato per la nostra analisi abbiamo esaminato l'uso del congiuntivo in italiano dopo espressioni appartenenti a due categorie: espressioni della modalità deontica, tra cui espressioni di significato volitivo, e espressioni della modalità epistemica, di significato cioè dubitativo e valutativo.

Con le espressioni della modalità epistemica, che esprimono un'opinione soggettiva, un dubbio o l'incertezza del parlante e che hanno un valore valutativo, prevale nettamente il congiuntivo (tabella $1 \mathrm{e}$ tabella 4). Risulta cioè che se il parlante può scegliere tra il congiuntivo e l'indicativo e decide di dare la preferenza al primo, vuol dire che lo sa usare con piena consapevolezza non solo per esprimere sfumature modali ma anche per esprimersi in modo più elegante $\mathrm{e}$ stilisticamente ricercato. Con le espressioni della modalità deontica viene usato in una proposizione dipendente quasi esclusivamente il congiuntivo (tabella 2). Abbiamo constatato una bassa frequenza della frase complessa con un'espressione modale nella frase principale, probabilmente perché la lingua parlata si distingue stilisticamente da quella scritta: parlando si preferisce una frase semplice a quella complessa. Dopo le espressioni modali che esprimono sensazioni prevale l'indicativo (tabella 3). Quindi si può concludere che la prevalenza del congiuntivo dopo i predicati che permettono anche l'uso dell'indicativo testimonia che esso continuerà a rimanere vivo anche nella lingua parlata. I dati ricavati dalla nostra 
analisi non possono dare un quadro completo della vitalità del congiuntivo nell'italiano parlato di oggi. Sono cioè solo un modesto contributo alla ricerca nel campo.

\section{BIBLIOGRAFIA}

Alisova Tatiana (1972), Strutture semantiche e sintattiche della proposizione semplice in italiano, Firenze, SGI.

BALLY Charles (1963), Linguistica generale e linguistica francese, Milano, Il Saggiatore.

Borgato Gianluigi; RENZI Lorenzo (1991), Tipi di frasi principali. Il tipo ottativo, in : ReNZI Lorenzo; SALVI Giampaolo; CARDINALETTI Anna (ed., 1991), p. 59-164.

BRonZI Anna Maria (1977), Indicativo e congiuntivo nelle completive italiane. SGI 6. Firenze, Accademia della Crusca, p. 425-471.

Della Valle Valeria; Patota Giuseppe (2009), Viva il congiuntivo! Come e quando usarlo senza sbagliare, Milano, Sperling and Kupfer.

FAVA Elisabetta (1978), Note su forme grammaticali e atti di domanda in italiano, Lingua e stile, XII, n. 1, p. 31-49.

FAVA Elisabetta (1991), Tipi di atti e tipi di frasi, in : RENZI Lorenzo, SALVI Giampaolo, CARDINALETTI Anna (ed., 1991), p. 19-48.

FAVA Elisabetta; SALVI Giampaolo (1991), Tipi di frasi principali. Il tipo dichiarativo in : RENZI Lorenzo, SALVI Giampaolo, CARDINALETTI Anna (ed., 1991), p. 49-69.

KLÍMOVÁ Eva (2006), Osservazioni sul valore modale del congiuntivo italiano, Sborník prací Ostravské univerzity, Studia Romanistica 6, Ostrava, p. 43-58.

KLÍMOVÁ Eva (2008), Modo e modalità della frase dipendente in italiano: dalla morfosintassi alla pragmatica, Rivista di Psicolinguistica Applicata, VIII/1-2, [KB 1787], p. 131-149.

LOMBARDI VALLAURI Edoardo (2003), Vitalità del congiuntivo nell'italiano parlato, in : Atti del XXXIV congresso della SLI, Roma, Bulzoni, p. 609-634.

MARAZZINI Claudio (2004), Breve storia della lingua italiana, Bologna, Il Mulino.

Molinelli Piera (1997), L'evoluzione del congiuntivo tra latino e romanzo: subordinate finali e consecutive, Linguistica e Filologia, 5, p. 179-194.

Palmer, F. R. (1986), Mood and Modality, Cambridge, Cambridge University Press.

RENZI Lorenzo; SALVI Giampaolo (ed., 1991), Grande grammatica italiana di consultazione, vol. II. Bologna, Il Mulino.

ReNZI Lorenzo; SAlvi Giampaolo; CARdinAletTI Anna (ed., 1991), Grande grammatica italiana di consultazione, vol. III. Bologna, Il Mulino.

SALVI Giampaolo; Borgato Gianluigi (1991), Tipi di frasi principali. Tipo iussivo, in: RENZI Lorenzo, SAlvi Giampaolo, CARDINALETTI Anna (ed., 1991), p. 152-159.

SCHNEIDER Stefan (1999), Il congiuntivo tra modalità e subordinazione, Roma, Carocci. 
SERIANNI Luca (1991), Grammatica italiana. Italiano comune e lingua letterari, Torino, UTET.

SIEROŃ Dorota (2013), Destinato a sparire? L'uso del congiuntivo a 150 anni dall'unità d'Italia sull'esempio de "La Repubblica". Uno studio corpus based, Romanica cracoviensia, 13, p. 86-93.

STATI Sorin (1982), Il dialogo. Considerazioni di linguistica pragmatica, Napoli, Liguori.

WANDRUSZKA Ulrich (1991), Frasi subordinate al congiuntivo, in : RENZI Lorenzo; SALVI Giampaolo (ed., 1991), p. 415-481. 